\title{
Macro-continuous Computed Torque Algorithm for a Three-dimensional Eel-like Robot
}

\author{
Frédéric Boyer, Mathieu Porez and Wisama Khalil (IEEE senior member)
}

\begin{abstract}
This paper presents the dynamic modeling of a continuous three-dimensional swimming eel-like robot. The modeling approach is based on the "geometrically exact beam theory" and on that of Newton-Euler, as it is well known within the robotics community. The proposed algorithm allows us to compute the robot's Galilean movement and the control torques as a function of the expected internal deformation of the eel's body.
\end{abstract}

Key words - Locomotion, biomimetic, eel-like robot, hyperredundant robot, Newton-Euler algorithms, Lie groups.

\section{INTRODUCTION}

$\mathrm{I}_{\mathrm{m}}^{\mathrm{n}}$ this paper, we will present the preliminary results of a multi-disciplinary research project supported by the French CNRS. The goal of the project is to design and control a three dimensional eel-like robot. As many authors working in the biomimetic robotics community have noted, eel-like robots present an interesting perspective for improving the efficiency and maneuverability of underwater vehicles [1-5]. The prototype we are designing will be a hyper-redundant robot made by connecting many parallel platforms. Moreover in order to guarantee efficient propulsion, it will be covered with a continuous deformable organ, which will mimic the eel's skin. This paper essentially deals with the macroscopic modeling of the future prototype. By "macroscopic" we mean a "high-level" model, which can be used as the basis for a preliminary design of the system and its control strategy. In particular, the macroscopic model does not take into account the detailed technology of the prototype but rather an ideal dynamic behavior useful to fix the guidelines of the project. The new results reported in this paper are multiple. Firstly, and contrary to most previous research on the same topic, the investigated robot is capable of three-dimensional swimming. Secondly, it is based on a continuous model adapted to the macroscopic modeling of the future hyperredundant prototype and to the continuous character of its skin. Some authors, using the concept of backbone curves

Manuscript received July 27, 2004. This work was supported in part by the French CNRS within the research programme "ROBEA".

Frédéric Boyer is with Institut de Recherche en Communications et Cybernétique de Nantes (IRCCyN, 1 rue de la Noë, B.P. 9210144321 Nantes cedex 3, France), école des Mines de Nantes (e-mail : frederic.boyer@emn.fr).

Mathieu Porez is with Institut de Recherche en Communications et Cybernétique de Nantes (IRCCyN), école centrale de Nantes (e-mail: mathieu.porez@irccyn.ec-nantes.fr).

Wisama Khalil is with Institut de Recherche en Communications et Cybernétique de Nantes (IRCCyN), école centrale de Nantes (e-mail: wisama.khalil@irccyn.ec-nantes.fr).
[6-8], have previously studied continuous modeling of hyper-redundant manipulators. In order to apply this kind of idea to the dynamics of the 3-dimensional framework, we adopted here the geometrically exact theory of beams in finite deformation, originally by J.C. Simo [9-11]. The idea consists in considering the eel robot as a beam defined by a continuous assembly of rigid cross sections and controlled through distributed laws of internal strains or torque. With this choice, just as in works dealing with articulated locomotion systems [12-14], the head dynamics of the continuous eel are derived on a fiber bundle. But in our case, while the fiber is still the group $S E(3)$ of the head displacements, the shape space is no longer a finite dimensional manifold but rather a functional space of curves in a Lie algebra. In fact, the shape space will be parameterized by the field of the infinitesimal transformations of the cross sections along the eel's backbone. Moreover, in accordance with the works of Simo, the eel's body dynamics will be written on the space of position-orientation of the beam cross sections with respect to the earth frame, i.e. a functional space of curves in a Lie group. However, contrary to the numerical approach proposed by Simo to integrate the dynamics of passive beams, the dynamics problem considered here is not solved with the standard numerical tools of nonlinear structural dynamics but using the "Newton-Euler philosophy" of rigid robotics [15-17]. Finally, the proposed approach turns out to be a generalization of the Newton-Euler based algorithm of Luh and Walker [15] applied to the case of a continuous robot with a mobile base (here imitating the eel's head). The algorithm gives the motion of the eel and the control torque evolution as outputs in terms of the deformation time-law of its body as inputs. As is well known from rigid robotics, the recursive nature of the Newton-Euler approach allows us to obtain efficient and fast algorithms, which are very simple to implement. Moreover, it gives us a straightforward link to the modeling of the future poly-articulated prototype. Finally, as far as the interaction of the fluid with the eel is concerned, for control requirements, we need to model the contact in a simple manner regarding the robustness of our future closed loop controllers. Two simple analytical models suited to our purposes exist. Both are based on the Fluid Mechanics' theory of the slender body [18]. The biomechanics community suggests the first one, the second is offered by the ocean-engineering community. The first is a result of the "Large amplitude elongated body theory of fish locomotion" by Lighthill [19]. This model is based on the basic assumption of the existence of some slices of fluid transversally transported with the cross sections of the eel. 
Then from kinetic conservation laws, the undulation of the eel's body generates the propulsion by reaction. Nevertheless, this model has been restricted until now to planar swimming. Hence, in order to investigate threedimensional swimming, we use the second model, which is, today, devoted to the dynamics of underwater flexible cables [20]. In this second model, like in Lighthill's planar solution, fluid forces are introduced through a local analytical model written for each transverse slice of the cable. Moreover this model takes into account, not only some inertial terms (like the Lighthill model does), but also some drag (transversal) and viscous (tangential) forces.

The paper is organized as follows: we will start by briefly presenting the relationships between nonlinear beam theory and hyper-redundant robots designed by the assembly of parallel platforms (section II). Then, some basic definitions and notations about the macroscopic beam model of the prototype are given (section III). Based on this model, the fluid-structure interaction is modeled (section IV). Section V is devoted to the continuous kinematic model of the eel. In section VI, we give the eel's head dynamics based on the principle of virtual works. In the Newton-Euler algorithm, these dynamics give the boundary conditions of the continuous kinematic models. Then the dynamic model of the eel's body is presented (section VII). It is based on the calculus of variations applied to a Cosserat medium [21], and leads us to a set of "partial differential equations" directly linked to those of Reissner's beam [22]. Finally, all these results are combined in section VIII in order to obtain the computed torque algorithm. The paper ends with numerical examples (section IX) and some concluding remarks (section $\mathrm{X})$.

\section{BEAM'S KINEMATICS AND HYPER-REDUNDANT ROBOTS}

We will consider here the case of a hyper-redundant robot produced by a serial assembly of parallel platforms. The robots investigated like so are of "snake-like" or "elephant trunk" type. In this context the rigid platforms mimic the vertebrae while the kinematics between any two platforms play the role of inter-vertebral kinematics. We develop the dynamics analysis using the geometrically exact theory of nonlinear beams, as developed in the eighties by J.C. Simo [9-11]. In this theory, the beam is considered as a continuous assembly of rigid sections of infinitesimal thickness, i.e. a one-dimensional Cosserat medium [21]. In the context of robotics, the sections imitate the robot's vertebrae and the beam kinematics, that of the inter-vertebral kinematics. Pushing the analogy forward, the centroidal line of the beam plays the role of the backbone of the snake-like robot. In this framework, one of the first tasks is to relate parallel platforms with the corresponding beam kinematics, where some of them are not related to any standard beam theory. In order to present these correspondences briefly, let us first introduce a few definitions related to the geometrically exact beam theory. Firstly, the material abscissa along the beam axis is denoted by $X$, which positions a particular cross section in a reference configuration $\Sigma_{o}$ (see Figure 1). The reference configuration is considered to be straight and represents a Galilean reference to which we fix the earth frame: $F_{g}=\left(O, E_{1}, E_{2}, E_{3}\right)$. Secondly, to each $X$ section, we materially fix a mobile ortho-normed frame $F_{X}=\left(G(X), t_{1}(X), t_{2}(X), t_{3}(X)\right)$, where $G(X)$ is the center mass of the $X$ section, and $t_{1}$ supports the beam axis $E_{1}$ when it is in the configuration $\Sigma_{o}$. Let us point out that $X \mapsto F_{X}$ is not the field of Frenet-Serret frames since each $F_{X}$ is actually attached to the $X$ cross section and not deduced from the geometry of the deformed backbone curve.

The advantage of this choice is that the torsion is no longer geometric but actually related to the torsion strain field of the beam. Now, let us introduce the rigid transformations of $S E(3)$ mapping the cross sections before deformation onto their configurations after deformation (defining the whole deformed configuration of the eel $\Sigma(t)$ (see Figure 1)):

$$
X \in[0,1] \mapsto g(X)=\left(\begin{array}{cc}
R(X) & d(X) \\
0 & 1
\end{array}\right)
$$

where $R$ is the rotation matrix mapping the $X$ mobile basis before deformation onto that after, and $d$ is the vector displacement field of the section centers. With the adopted parametrization (1), the deformation of the beam is defined by the field of twist $X \in[0,1] \mapsto \eta(X) \in \operatorname{se}(3)$ modeling the infinitesimal changes of the mobile frames situation when sliding along the beam's material line of centroids, i.e., s.t.:

$$
\partial g / \partial X=g \cdot \eta
$$

which can be expressed as:

$$
\left(\begin{array}{cc}
\partial R / \partial X & \partial d / \partial X \\
0 & 0
\end{array}\right)=\left(\begin{array}{ll}
R & d \\
0 & 1
\end{array}\right) \cdot\left(\begin{array}{cc}
\hat{K} & \Gamma \\
0 & 0
\end{array}\right)
$$

where we introduced the skew-symmetric tensor: $\hat{K}=R^{T}(\partial R / \partial X)$ associated to the axial vector $K$. The last two components $K_{2}$ and $K_{3}$ of $K$ stand for the curvatures of the beam in the two planes $\left(G, t_{1}, t_{3}\right)(X)$ and $\left(G, t_{1}, t_{2}\right)(X)$, while $K_{1}$ is the rate (per unit of material length) of rotation of the section around its normal vector, i.e. the torsion strain field. As for the infinitesimal translations between two sections, we introduced the vector $\Gamma=R^{T}(\partial d / \partial X)$, whose first component $\Gamma_{1}$ is related to the stretching of the beam while the two others are related to its transverse shearing [11]. An eel-like robot can then be considered as a beam controlled by a desired time evolution of $\eta$ : $t \mapsto \eta_{d}(X, t), \forall X$, under the assumption that local controllers are able to impose the desired strains $\Gamma$ and $K$ instantaneously. We shall see later, in the particular case of our eel-like robot, how to relate the strain law $(K, \Gamma)$ with that of the motor torques. Now, let us relate the general beam kinematics (2)-(3) to the corresponding hyperredundant robots.

To do this, we shall introduce a few possible parallel structures, starting from the most general case to the most specific one, by constraining the time evolution of $\eta$ more 
and more. In the most general case, all six components of the vectors $K$ and $\Gamma$ can be defined by some arbitrary time evolutions. The corresponding parallel platform is of "Gough-Stewart type" [23], and the beam kinematics is that of Timoshenko-Reissner [22,24]. Another interesting case consists in imposing $\Gamma_{2}=\Gamma_{3}=0$, in this case the intervertebral kinematics allows a three d.o.f. rotation and stretching along the backbone. The corresponding continuous model is that of an extensible Kirchoff beam [25]. The next interesting kinematics is deduced by imposing $\Gamma_{1}=1$. In this case we have $\partial r / \partial X=t_{1}$ (where $r$ is the position field of the beam axis (see Figure 1)), and the Kirchoff beam is non-stretchable while the corresponding parallel platform is of "spherical joint type". Finally for the purpose of three-dimensional manipulation or locomotion, the minimal kinematics consists in imposing $K_{1}=0$, in this case the robot cannot twist around its backbone, nevertheless it can roll around it, by combining the two bending curvatures $K_{2}$ and $K_{3}$. In this case, the platform is of "universal joint type". If we go further, by imposing $K_{2}=0$ the robot is a planar one as those designed for planar swimming in [4]

Finally, note that the constraints imposed by robot architecture design can be interpreted as specific time evolutions, and that from a dynamics point of view, these constraints will induce internal reaction forces and torque fields playing the role of Lagrange multipliers. In the same manner, time evolution of the internal d.o.f. will be imposed by the corresponding internal control torques and forces. This point will be discussed at the end of the paper taking case of our eel-like robot.

\section{BASIC DEFINITIONS AND NOTATIONS}

First and foremost, note that throughout the paper, any tensor fields depend on time and the space variable $X$. Moreover, time dependency can be explicit when the fields are known through their time evolution, or implicit, if their time evolutions require us to integrate the dynamics. In the first case, time appears explicitly as an argument of the field, but not in the second case. Finally the derivative operators " $\partial . / \partial X$ " and " $\partial . / \partial t$ " will be indicated by a "prime" and a "dot" respectively. In this section, we will give all the geometric and inertia characteristics of the macroscopic model of the prototype. Firstly we will introduce the following basic definitions from the continuous approach of the geometrically exact beam theory.

\section{A. Basic definitions}

In accordance with Figure 1, we will use the following frames:

- The Galilean frame (fixed to the earth), written as $F_{g}=\left(O, E_{1}, E_{2}, E_{3}\right)$,

- The mobile frame of the section (vertebra) $X$ of the eel's body, indicated as $F_{X}=\left(G(X), t_{1}(X), t_{2}(X), t_{3}(X)\right)$,
- The mobile frame of the eel's head, which is written as $F_{o}=\left(O_{o}, t_{1 o}, t_{2 o}, t_{3 o}\right)$, (it is the mobile frame of the section $X=0$ of the body).

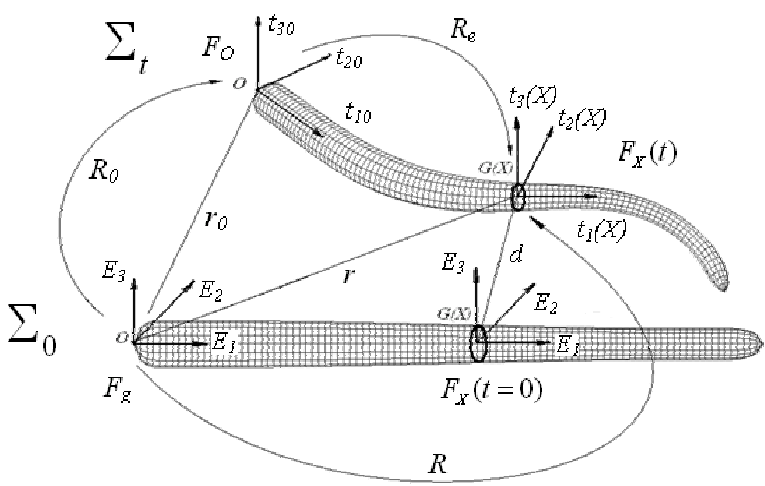

Figure 1: Frames and parameterisation.

With these three sets of frames we define the set of curves, which defines the configuration space of the eel (summation convention on repeated indexes is adopted):

$$
\begin{aligned}
& {[0,1] \rightarrow \mathbb{R}^{3} \times S O(3)} \\
& X \mapsto\left(r(X)=r_{i}(X) E_{i}, R(X)=t_{i}(X) \otimes E_{i}\right)
\end{aligned}
$$

where " $\otimes$ " is the tensor product.

In the following we will note more simply:

$$
R(X=0)=R_{o}, \quad r(X=0)=r_{o}
$$

which describe the orientation and position of the head in relation to the earth.

\section{B. Geometric and inertial characteristics of the eel-like} robot

We suppose that a continuous beam of rigid elliptic shape cross sections represents the prototype. The mass distribution is supposed to be homogeneous and of unit density. Using these assumptions, the center of mass of any section coincides with the geometric center of the ellipse. For the same reasons, the inertia principal axes of the inertia tensor density along the beam coincide with the ellipse axes. Thus, taking the mobile vectors basis $t_{2}$ and $t_{3}$ aligned with the small and great ellipse axis respectively, forces the inertia density to be as follows:

$$
d I / d X=\rho J_{1} t_{1} \otimes t_{1}+\rho J_{2} t_{2} \otimes t_{2}+\rho J_{3} t_{3} \otimes t_{3}
$$

where $\rho$ is the mass per unit of volume of the material and $J_{i}, i=1,2,3$ are the geometric second order moments of the section around $t_{i}, i=1,2,3$. Finally, with these assumptions, the centers of mass and buoyancy are collocated and the whole robot is neutrally buoyant. This means that the simulated prototype is assumed to be able to control its density to allow neutral buoyancy at all depths. 


\section{Inter-vertebral kinematics}

Based on an analysis of three-dimensional swimming of fish, we adopted a spherical inter-vertebral kinematics. With this choice, the corresponding beam theory is that of nonstretchable Kirchoff beams [25], while the parallel platforms are of "spherical joint type". Thus, the control inputs of the dynamics problem is the time evolution of the twistcurvature tensor field:

$$
\forall X, \hat{K}_{d}(X, t)=R^{T}(\partial R / \partial X)=R^{T} R^{\prime}
$$

In the following, for any $V \in \mathbb{R}^{3}, \hat{V}$ will denote the skewsymmetric tensor s.t.: $\forall U \in \mathbb{R}^{3}, \hat{V} U=V \times U, \quad$ and conversely for a skew-symmetric tensor $\xi$ of $s o(3), \xi^{\vee}$ will denote the associated pseudo-vector of $\mathbb{R}^{3}$ s.t. $\xi V=\xi^{\vee} \times V$. Finally, the "spherical kinematics" imposes the mechanical design constraint:

$$
\partial r / \partial X=r^{\prime}=t_{1}
$$

which forces the beam to verify the Kirchoff and nonextensibility assumptions.

\section{FLUID-STRUCTURE INTERACTION MODEL}

To model the contact forces between the fluid and the eel's body, we will adopt a standard generalization of the Morison model [26] applied to the case of under-water flexible cables of circular cross section [20]. This model takes into account not only the transverse reactive (inertial) forces like Lighthill's model [19], but also the resistive (viscous) tangential ones. However, it excludes the influence of the vortexes shaded in the eel's wake. In order to apply this model to the case of the eel, we added a slight modification to it that takes into account the elliptic profile of the eel's sections (see Appendix). In this model, based on the "slender body approximation" of Fluid Mechanics [18], the fluid forces are modeled "slice-by-slice" through the field of (left invariant) wrench density along the eel's backbone $\left(s o(3)^{*}\right.$ is the dual space of the Lie algebra of $S O(3)$, i.e. the space of torques):

with:

$$
] 0,1\left[\rightarrow \mathbb{R}^{3} \times \operatorname{so}(3)^{*}, \quad X \mapsto\left(d f_{\text {ext }} / d X, d c_{\text {ext }} / d X\right),\right.
$$

$$
d f_{e x t} / d X=-C_{l d 1}\left|V_{1}\right| V_{1} t_{1}-\sum_{i=2}^{3} C_{l d i}\left\|v_{n}\right\| V_{i} t_{i}-\sum_{i=2}^{3} C_{l m i} \Upsilon_{i} t_{i}
$$

where we introduced the velocity and acceleration of each section mass center in the corresponding section frame:

$$
\dot{r}=\sum_{i=1}^{3} V_{i} t_{i}=V_{1} t_{1}+v_{n}, \ddot{r}=\sum_{i=1}^{3} \Upsilon_{i} t_{i}
$$

with $v_{n}$, the section velocity normal to the eel axis, and $C_{l d i}$ and $C_{l m i}$, are coefficients depending on the mass per unit of volume of the fluid, the shape and size of the profile (here elliptic) and the Reynolds number of the moving profile in the fluid. At this point, it is worth noting that the local model (9) generates some drag and lift resultant forces on the global eel dynamics. For instance, let us consider the simplified case where the eel is straight (rigidified) and fixed at rest in a steady flow of velocity $v$ with an angle of attack $\alpha$ (see Figure 2). In these conditions ( $\dot{r}=-v E_{1}, \ddot{r}=0$ ), the resultant of local forces (9) turns out to be after computations:

$$
F=\int_{0}^{1}\left(d f_{e x t} / d X\right) d X=-C_{d} v^{2} E_{1}-C_{l} v^{2} E_{3}
$$

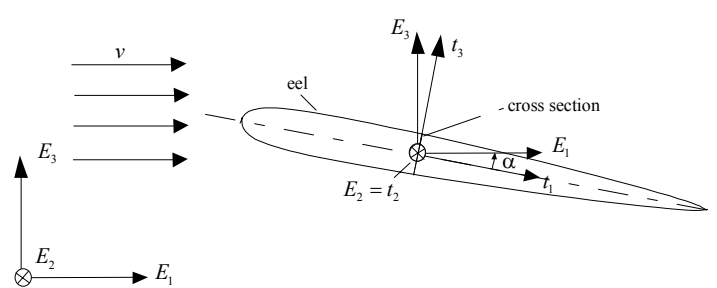

Figure 2: Eel in a straight rigidified configuration fixed in a steady flow.

where $C_{d}$ and $C_{l}$ are respectively two $\alpha$ dependent drag and lift coefficients, expressed as:

$$
\begin{gathered}
C_{d}=-\int_{0}^{1}\left(C_{l d 1}|\mathrm{c} \alpha| \mathrm{c}^{2} \alpha+C_{l d 3}|\mathrm{~s} \alpha| \mathrm{s}^{2} \alpha\right) d X \\
C_{l}=\int_{0}^{1}\left(C_{l d 1}|\mathrm{c} \alpha|-C_{l d 3}|\mathrm{~s} \alpha|\right) \mathrm{s} \alpha \mathrm{c} \alpha d X
\end{gathered}
$$

Note that these expressions of global drag and lift coefficients are approximations within the slender body theory, which do not take into account the influence of the eel's wake. However contrarily to the Lighthill model [19], they take into account tangential viscous resistance as it is shown by imposing $\alpha=0$ in $C_{d}$. Finally, the acceleration terms of (9) are the added mass forces of [20] as they are extended to elliptic cross sections in [27].

As far as the field of fluid torque is concerned, we take into account the drag and added inertia torques generated by the rotation of the planar elliptic cross sections around their normal axis:

$$
d c_{e x t} / d X=-C_{a d 1}\left|\Omega_{1}\right| \Omega_{1} t_{1}-C_{a m 1} \dot{\Omega}_{1} t_{1}
$$

where we introduced the angular velocity and acceleration of the section in its mobile frame:

$$
\omega=\Omega_{i} t_{i} \quad, \quad \dot{\omega}=\dot{\Omega}_{i} t_{i}
$$

We can summarize all these terms by the following contact law:

$$
\left(\begin{array}{l}
d f_{\text {ext }} / d X \\
d c_{\text {ext }} / d X
\end{array}\right)=\left(\begin{array}{l}
d f_{d r a g} / d X \\
d c_{d r a g} / d X
\end{array}\right)-\left(\begin{array}{cc}
c_{l m} & 0 \\
0 & c_{a m}
\end{array}\right) \cdot\left(\begin{array}{l}
\ddot{r} \\
\dot{\omega}
\end{array}\right)
$$

where we introduced the density of drag wrench, which generates some global drag and lift (see the previous simplified example): 


$$
\left(\begin{array}{c}
d f_{d r a g} / d X \\
d c_{d r a g} / d X
\end{array}\right)=\left(\begin{array}{c}
-C_{l d 1}\left|V_{1}\right| V_{1} t_{1}-\sum_{i=2}^{3} C_{l d i}\left\|v_{n}\right\| V_{i} t_{i} \\
-C_{a d 1}\left|\Omega_{1}\right| \Omega_{1} t_{1}
\end{array}\right)
$$

and also the sectional added mass tensors depending on the geometry of the section profile and the fluid characteristics:

$$
c_{l m}=C_{l m 2} t_{2} \otimes t_{2}+C_{l m 3} t_{3} \otimes t_{3}, c_{a m}=C_{a m 1} t_{1} \otimes t_{1}
$$

where with an $X$ dependent profile of sections (as in our case), the parameters of (15) will vary along the eel's backbone. It is worth noting that as in the case of lift and drag resultant forces, the added mass contributions of (13) will be superimposed slice-by-slice. For instance, if we consider the case where the eel is a rigid ellipsoid (where the lengths of its three axis supported by $t_{1}, t_{2}$ and $t_{3}$ are respectively $2 A, 2 B, 2 C$ ), then the resultant of all the sliceby-slice added mass contributions when calculated at the ellipsoid center mass $G$, can be broken down as the following $6 \times 6$ matrix in the eel's frame centered on $G$ :

$$
\left(\begin{array}{cc}
m_{a} & m \hat{s}_{a}^{T} \\
m \hat{s}_{a} & I_{a}
\end{array}\right)_{G}=\left(\begin{array}{cc}
\operatorname{diag}\left(m_{a i}\right) & 0 \\
0 & \operatorname{diag}\left(I_{a i}\right)
\end{array}\right),
$$

where with standard expressions of $C_{l m 2}, C_{l m 3}$ and $C_{a m 1}$ for an elliptic section, (given later in section IX.A): $m_{a 1}=0$, $m_{a 2}=4 \rho \pi A C^{2} / 3, \quad m_{a 3}=4 \rho \pi A B^{2} / 3, \quad I_{a 3}=4 \rho \pi C^{2} A^{3} / 15$, $I_{a 2}=4 \rho \pi B^{2} A^{3} / 15$, and $I_{a 1}=2 \rho \pi\left(B^{2}-C^{2}\right)^{2} A / 15$. Finally note that these expressions are nothing else than those of the added masses and inertia of a general ellipsoid [28] where $B / A$ and $C / A \rightarrow 0$, i.e. within the "slender body theory". In the case of the eel, this approximation essentially models the reactive transverse fluid forces on which undulatory swimming is based [19].

In order to complete this approximated model let us now consider the forces applied to the two ends of the eel, i.e. its nose and its tail. Because the nose, i.e. section $X=0$ is geometrically reduced to a material point, we only take into account some dragging and added mass forces onto the head aligned with its axis, i.e.:

$$
f_{d, \text { head }}=-C_{d, \text { head }} V_{1}(0)\left|V_{1}(0)\right| t_{1 o}, f_{m, \text { head }}=-C_{m, \text { head }} \Upsilon_{1}(0) t_{1 o}
$$

where the parameters $C_{d, \text { head }}$ and $C_{m, \text { head }}$ depend on the shape of the head. Finally, because the wake influence is neglected, the wrench applied to the terminal section $X=1$ is assumed to be zero.

\section{CONTINUOUS KINEMATIC MODEL OF THE EEL}

In this section, we develop the continuous version of the kinematic models of open robotic chains. All these models can be interpreted as p.d.e.s in space and time. Nevertheless, when we deal with the dynamic algorithm, these p.d.e.s will be interpreted as spatial o.d.e.s and numerically integrated at each step in a time loop.

\section{A. Continuous geometric model}

From (7) and (8) we obtain the two following models.

Model of orientations:

$$
R^{\prime}=R \hat{K}_{d} \quad, \quad R(0)=R_{o}
$$

Model of position:

$$
r^{\prime}=t_{1}, \quad r(0)=r_{o}
$$

\section{B. Continuous Kinematic model of velocity}

\section{Model of angular velocity:}

Time-differentiating (17) and introducing the field of angular velocities:

$$
\begin{aligned}
& \hat{\omega}:[0,1] \rightarrow \operatorname{so}(3) \\
& X \mapsto \dot{R}(X) R(X)^{T}=\hat{\omega}(X)
\end{aligned}
$$

we find (see [29] for more details about the demonstration):

$$
\hat{\omega}^{\prime}=\dot{\hat{k}}+[\hat{k}, \hat{\omega}]
$$

with $\hat{k}=R \hat{K}_{d} R^{T}$ and [.,.], the Lie bracket of so(3). Finally, we can rewrite (20) in terms of axial vectors as:

$$
\omega^{\prime}=\dot{k}+k \times \omega=R \dot{K}_{d}
$$

with the boundary condition given by the eel's head angular velocity $\omega(X=0)=\omega_{o}=\left(\dot{R}_{o} R_{o}^{T}\right)^{\vee}$.

\section{Model of linear velocity:}

By time-differentiating (18):

$$
\dot{r}^{\prime}=\omega \times t_{1}
$$

with the boundary condition given by: $\dot{r}(X=0)=\dot{r}_{o}$.

\section{Continuous kinematic model of acceleration:}

Time-differentiating (21) and (22), gives the two following models.

\section{Model of angular acceleration:}

$$
\begin{gathered}
\dot{\omega}^{\prime}=\ddot{k}+\dot{k} \times \omega+k \times \dot{\omega}=R \ddot{K}_{d}+\omega \times R \dot{K}_{d}, \\
\dot{\omega}(X=0)=\dot{\omega}_{o}=\left(\ddot{R}_{o} \cdot R_{o}^{T}+\dot{R}_{o} \cdot \dot{R}_{o}^{T}\right)^{\vee}
\end{gathered}
$$

Model of linear acceleration:

$$
\ddot{r}^{\prime}=\dot{\omega} \times t_{1}+\omega \times\left(\omega \times t_{1}\right), \quad \ddot{r}(X=0)=\ddot{r}_{o}
$$

Let us note that (23) and (24) are two p.d.e.s whose space integration at each step of time will give the acceleration field along the eel's backbone. Thus, they represent two o.d.e.s, which are the continuous counterparts of the forward recurrences of acceleration of a multibody system, where $X$ replaces the body index. Nevertheless, contrary to the case of manipulators, (23) and (24) are not initialized by some imposed boundary conditions but rather by the acceleration 
of the eel's head. Hence, we have to derive the head's dynamics in order to compute (at each step in time) the head's acceleration (see section VI).

\section{Decomposition of acceleration}

As we shall see in the next section, the computation of the head's dynamics requires us to break down acceleration along the eel's body into two components, one depending on the head's acceleration, the other containing the terms relative to velocity and internal strain (curvature and twist) acceleration. Such decomposition is based on the following parametrization of the eel's configuration:

$$
\begin{aligned}
& R(X)=R_{o} R_{e}(X) \\
& r(X)=r_{o}+r_{e}(X)
\end{aligned}
$$

where $R_{e}(X)$ and $r_{e}(X)$ are the orientation and position of the $X$ section respectively, in relation to the head frame. Finally, the spatial differentiation of (25) and (26) shows that $R_{e}$ and $r_{e}$ are, at any time, the solutions of (17) and (18) , with $R(X=0)=1$ and $r(X=0)=0$.

\section{Decomposition of angular acceleration}

If we insert (25) into (19), we find, after time differentiation:

$$
\dot{\omega}(X)=\dot{\omega}_{o}+\dot{\omega}_{e}(X)
$$

where $\dot{\omega}_{o}$ is the angular acceleration of the head and $\dot{\omega}_{e}$ is the solution of the following p.d.e. obtained by spatial differentiation of (27):

$$
\dot{\omega}_{e}^{\prime}=\dot{\omega}^{\prime}=\ddot{k}+\dot{k} \times \omega+k \times \dot{\omega}
$$

and so, $\dot{\omega}_{e}$ is simply the solution of (23), with $\dot{\omega}(X=0)=0$.

\section{Decomposition of linear acceleration}

Now inserting (26) into the definition of $\ddot{r}$ leads us to the introduction of a new acceleration term $\gamma_{e}$ defined by:

$$
\ddot{r}=\ddot{r}_{o}+\dot{\omega}_{o} \times r_{e}+\gamma_{e}
$$

Then, differentiating (29) with respect to the space variable, and taking (24) into account with the fact that $r_{e}^{\prime}=r^{\prime}=t_{1}$, we find:

$$
\left.\gamma_{e}^{\prime}=\dot{\omega}_{e} \times t_{1}+\omega \times\left(\omega \times t_{1}\right)\right)
$$

Thus, $\gamma_{e}$ is the solution of (24) with the boundary condition $\ddot{r}(X=0)=0$, and with $\dot{\omega}$ replaced by $\dot{\omega}_{e}$. Finally, the decomposition of acceleration (27) and (29) can be summarized by the following matrix relation:

$$
\left(\begin{array}{c}
\ddot{r} \\
\dot{\omega}
\end{array}\right)=\left(\begin{array}{cc}
1 & -\hat{r}_{e} \\
0 & 1
\end{array}\right)\left(\begin{array}{l}
\ddot{r}_{o} \\
\dot{\omega}_{o}
\end{array}\right)+\left(\begin{array}{l}
\gamma_{e} \\
\dot{\omega}_{e}
\end{array}\right)
$$

Hence, from (31) we see that $\gamma_{e}$ and $\dot{\omega}_{e}$ are two acceleration terms containing relative acceleration due to the deformation of the body and all the Coriolis-centrifugal terms.

\section{THE EEL'S HEAD'S DYNAMICS}

In this section we adopt the following definition of the eel configuration space: $\mathfrak{C}:=S E(3) \times\{\hat{K}:[0,1] \mapsto s o(3)\}$, i.e. a principal fiber bundle with $S E(3)$ the Euclidean head displacement and $\{\hat{K}:[0,1] \mapsto s o(3)\}$, the shape space of twist-curvatures along the beam. The dynamics of the head are easily derived from the principle of virtual works:

$$
\delta W_{d y n}=\delta W_{e x t}, \forall \delta \phi, \delta r
$$

where $\delta \phi=\left(\delta R R^{T}\right)^{\vee}$ and $\delta r$ are the virtual angular and linear displacement fields applied to the cross sections while the time is maintained. The left-hand side of (32) represents the virtual work of acceleration quantities while its right counterpart is the virtual work of external forces here due only to the contact with fluid. Note that the control torque field does not produce any virtual work, since at this point, the internal deformations are directly imposed through the time strain law (7). Applying this principle with (31) and the following form of the virtual displacement field:

$$
\left(\begin{array}{c}
\delta r \\
\delta \phi
\end{array}\right)=\left(\begin{array}{cc}
1 & -\hat{r}_{e} \\
0 & 1
\end{array}\right) \cdot\left(\begin{array}{c}
\delta r_{o} \\
\delta \phi_{o}
\end{array}\right)
$$

which is compatible with (31) and with the "fixed time constraint" imposed by the application of the principle [30]. Finally, we obtain the eel's head's dynamics:

$$
\begin{aligned}
&\left(\left(\begin{array}{cc}
m 1 & m \hat{s}^{T} \\
m \hat{s} & I
\end{array}\right)+\left(\begin{array}{cc}
m_{a} & m \hat{s}_{a}^{T} \\
m \hat{s}_{a} & I_{a}
\end{array}\right)\right)\left(\begin{array}{c}
\ddot{r}_{o} \\
\dot{\omega}_{o}
\end{array}\right) \\
&+\left(\left(\begin{array}{l}
f_{e} \\
c_{e}
\end{array}\right)-\left(\begin{array}{l}
f_{e a} \\
c_{e a}
\end{array}\right)\right)=\left(\begin{array}{l}
f_{d l} \\
c_{d l}
\end{array}\right)
\end{aligned}
$$

which correspond to the Newton-Euler equations of the whole robot driven by the forced deformations. In (34) we introduced the following definitions:

the inertia tensor:

$$
\left(\begin{array}{cc}
m 1 & m \hat{s}^{T} \\
m \hat{s} & I
\end{array}\right)=\int_{0}^{1}\left(\begin{array}{cc}
\rho A 1 & -\rho A \hat{r}_{e} \\
\rho A \hat{r}_{e} & \rho I+\rho A \hat{r}_{e}^{T} \hat{r}_{e}
\end{array}\right) d X
$$

the wrench produced by Coriolis-centrifugal and strain acceleration:

$$
\left(\begin{array}{l}
f_{e} \\
c_{e}
\end{array}\right)=\int_{0}^{1}\left(\begin{array}{cc}
1 & 0 \\
\hat{r}_{e} & 1
\end{array}\right)\left(\begin{array}{c}
\rho A \gamma_{e} \\
\rho I \dot{\omega}_{e}+\omega \times(\rho I \omega)
\end{array}\right) d X
$$

the added mass tensor induced by the head acceleration: 


$$
\begin{aligned}
\left(\begin{array}{cc}
m_{a} & m \hat{s}_{a}^{T} \\
m \hat{s}_{a} & I_{a}
\end{array}\right) & =\int_{0}^{1}\left(\begin{array}{cc}
c_{l m} & -c_{l m} \hat{r}_{e} \\
\hat{r}_{e} c_{l m} & c_{a m}-\hat{r}_{e} c_{l m} \hat{r}_{e}
\end{array}\right) d X \\
& +\left(\begin{array}{cc}
c_{l m, \text { head }} & 0 \\
0 & 0
\end{array}\right)
\end{aligned}
$$

the wrench produced by added mass submitted to Coriolis, centrifugal, and strain acceleration:

$$
\left(\begin{array}{l}
f_{e a} \\
c_{e a}
\end{array}\right)=\int_{0}^{1}\left(\begin{array}{cc}
1 & 0 \\
\hat{r}_{e} & 1
\end{array}\right)\left(\begin{array}{c}
c_{l m} \gamma_{e} \\
c_{a m} \dot{\omega}_{e}
\end{array}\right) d X
$$

the wrench of drag and lift forces generated by the density of local drag wrench:

$$
\left(\begin{array}{l}
f_{d l} \\
c_{d l}
\end{array}\right)=\int_{0}^{1}\left(\begin{array}{ll}
1 & 0 \\
\hat{r}_{e} & 1
\end{array}\right)\left(\begin{array}{l}
d f_{\text {drag }} / d X \\
d c_{d r a g} / d X
\end{array}\right) d X+\left(\begin{array}{c}
f_{d, \text { head }} \\
0
\end{array}\right)
$$

\section{Remark: "body deformation dependency"}

Note that (35)-(39) take into account the influence of body deformation. For instance, transforming the local drag wrench from the $X$ cross section to the eel's head $(X=0)$ produces the term under the integral of (39). Hence, (39) first depends on body deformation through the relative position vector $r_{e}=r-r_{o}$. Moreover, (35)-(39) are intrinsic equations, i.e. are not projected in any frame. For instance, projecting (39) in the eel's head frame (this is what is done for numerical purpose), we have:

$$
\left(\begin{array}{c}
{ }^{o} f_{d l} \\
{ }^{o} c_{d l}
\end{array}\right)=\int_{0}^{1}\left(\begin{array}{cc}
R_{e} & 0 \\
{ }^{o} \hat{r}_{e} R_{e} & R_{e}
\end{array}\right)\left(\begin{array}{c}
X \\
\left(d f_{\text {drag }} / d X\right) \\
{ }^{X}\left(d c_{\text {drag }} / d X\right)
\end{array}\right) d X+\left(\begin{array}{c}
{ }^{o} f_{d, \text { head }} \\
0
\end{array}\right),
$$

where ${ }^{x} T$ denotes the matrix of the components of the tensor $T$ in the mobile base of the $X$ cross section. In particular, ${ }^{\circ} T$ is the matrix of the components of $T$ in the mobile basis of the section $X=0$, i.e. in the vector basis of the eel's head. Hence, the resultants (35)-(39) also depend on body deformation through the orientation field $R_{e}$. Finally, it can be noted that except the basic assumptions about the local contact model, no simplification is made in (35)-(39). These are the characteristics of the geometric exact theory. For instance, plunging the eel into the void and imposing some time varying deformations should conserve six "first integrals", i.e. the components of the linear and angular moments in the earth frame. This has been verified on the simulator with a precision of $10^{-6}$.

\section{THE DYNAMICS OF THE EEL'S BODY}

In this section we adopt the following definition of the eel configuration space: $\mathfrak{C}^{\mathfrak{x}}:=\left\{(r, R):[0,1] \mapsto \mathbb{R}^{3} \times S O(3)\right\}$, i.e. a space of curves in a Lie group. We have previously supposed that the internal strains were instantaneously imposed on the vertebrae through their time evolutions (7). In order to obtain the time control torque law required to verify this evolution, we are now going to derive the dynamics of internal forces and momentum. This can be done in a straightforward manner from a variational calculus applied to the following augmented Lagrangian:

$$
L=\int_{0}^{1} \frac{1}{2}\left(\dot{r}^{T}, \omega^{T}\right)\left(\begin{array}{c}
\rho A \dot{r} \\
\rho I \omega
\end{array}\right)+\left(\left(r^{\prime}-t_{1}\right)^{T},\left(K-K_{d}\right)^{T}\right)\left(\begin{array}{c}
n \\
M
\end{array}\right) d X
$$

where the first term stands for the kinetic energy of the eel; the second takes into account the constraints imposed to the inter-vertebral kinematics by the design (8) and the control (7). In order to force these constraints, we introduced the following field:

$$
\begin{aligned}
& {[0,1] \rightarrow \mathbb{R}^{3} \times \operatorname{so}(3)^{*}} \\
& X \mapsto\left(n(X)^{T}, M(X)^{T}\right)^{T},
\end{aligned}
$$

where, $n$ is the field of internal forces in the earth frame and $M$ is the field of control torque in the vertebra (cross section) frames. Then posing the "extended Hamilton principle" [31]:

$$
\delta \int_{t_{i}}^{t_{f}} L d t=\int_{t_{i}}^{t_{f}} \delta W_{e x t} d t, \forall \delta r, \delta \phi, \delta n, \delta M
$$

where the external contribution is due to the fluid (13), we obtain the partial differential equations of the eel's body which represent the Newton-Euler equations of each section:

$$
\begin{aligned}
& \forall X \in] 0,1[: \\
& \left(\begin{array}{c}
\left(\rho A-c_{l m}\right) \ddot{r} \\
\left(\rho I-c_{a m}\right) \dot{\omega}+\omega \times(\rho I \omega)
\end{array}\right)+\left(\begin{array}{c}
n^{\prime} \\
(R M)^{\prime}+t_{1} \times n
\end{array}\right)=\left(\begin{array}{c}
d f_{\text {drag }} / d X \\
d c_{\text {drag }} / d X
\end{array}\right)
\end{aligned}
$$

which has to be completed with the constraints:

$$
\forall X, K(X)=K_{d}(X, t) \quad, \quad r^{\prime}-t_{1}=0
$$

and the boundary conditions on the head given by (16):

$$
\left(\begin{array}{c}
n(0) \\
M(0)
\end{array}\right)=\left(\begin{array}{c}
f_{d, \text { head }}+c_{l m, \text { head }} \ddot{r}_{o} \\
0
\end{array}\right)
$$

and on the tail:

$$
\left(\begin{array}{c}
n(1) \\
M(1)
\end{array}\right)=\left(\begin{array}{l}
0 \\
0
\end{array}\right)
$$

Finally, before detailing our algorithm, let us note that the set of equations (42)-(45) defines a closed formulation enabling us to solve the direct dynamic problem of the eel, i.e. to compute the motion of the sections based on the knowledge of the internal torque law. This could be achieved for instance through the geometrically exact approach of finite elements as proposed in [11]. Instead, this set of equations will be used to solve the inverse problem, consisting in computing the internal control torque law corresponding to some expected deformation. In this case, we shall use (42) as spatial o.d.e.s integrated at each step of a simulation time loop. Let us note that in such a case, only the equations (42) with one of the two boundary conditions (44)-(45) are required since the constraints (43) are implicitly taken into account through continuous kinematics, 
while the second of the two boundary conditions is taken into account via the eel's head dynamics.

\section{COMPUTATIONAL ALGORITHM}

Based on the previous results, we can derive an algorithm whose inputs are the state variables of the eel's head, and the desired time evolution of its body's curvature and twist (and their time derivatives), i.e.:

and:

$$
\left(r_{o}, \dot{r}_{o}, R_{o}, \omega_{o}\right),
$$

$$
\forall X, \quad t \mapsto\left(K_{d}(X, t), \dot{K}_{d}(X, t), \ddot{K}_{d}(X, t)\right)
$$

The outputs of the algorithm are head acceleration and the required control torque for imposing (46), i.e.:

$$
t \mapsto\left(\ddot{r}_{o}(t), \dot{\omega}_{o}(t), M_{d}(X, t) \forall X\right)
$$

The algorithm is based on three integration numerical loops, the first one is in time and allows us to update the state of the head, and the two others are in space and are included in the first. The first space integration loop computes head acceleration. The second space integration loop calculates the control torque distribution applied to the vertebrae.

\section{A. First space integration loop}

The first space loop starts with the following spatial o.d.e.'s deduced from the p.d.e.'s of section V, now considered at the fixed current time $t$ of the time loop.

\section{Computation of the configuration}

- In orientation:

$$
R^{\prime}=R \hat{K}_{d}, R(0)=R_{o}
$$

- in position:

$$
r^{\prime}=R E_{1}, r(0)=r_{o}
$$

\section{Computation of the velocities}

- In orientation:

$$
\omega^{\prime}=R \dot{K}_{d} \quad, \omega(0)=\omega_{o}
$$

- in position:

$$
\dot{r}^{\prime}=\omega \times r^{\prime}, \quad \dot{r}(0)=\dot{r}_{o}
$$

\section{Computation of the acceleration:}

- In orientation:

$$
\dot{\omega}_{e}^{\prime}=R \ddot{K}_{d}+\omega \times R \dot{K}_{d}, \dot{\omega}_{e}(0)=0
$$

- In position:

$$
\gamma_{e}^{\prime}=\dot{\omega}_{e} \times r^{\prime}+\omega \times\left(\omega \times r^{\prime}\right), \gamma_{e}(0)=0
$$

\section{Computation of the head dynamics:}

Let us note that the tensors and wrenches of (34) can be computed at each step of the time loop by integrating the following system of o.d.e.'s with respect to the space variable from the head to the tail:

$$
\begin{gathered}
\mathbb{I}^{\prime}=\frac{d}{d X}\left[\left(\begin{array}{cc}
m 1_{3} & m \hat{s}^{T} \\
m \hat{s} & I
\end{array}\right)+\left(\begin{array}{cc}
m_{a} & m \hat{s}_{a}^{T} \\
m \hat{s}_{a} & I_{a}
\end{array}\right)\right] \\
=\left(\begin{array}{cc}
\rho A 1+c_{l m} & -\left(\rho A 1+c_{l m}\right) \hat{r}_{e} \\
\hat{r}_{e}\left(\rho A 1+c_{l m}\right) & \rho I+c_{a m}-\hat{r}_{e}\left(\rho A 1+c_{l m}\right) \hat{r}_{e}
\end{array}\right)(54),
\end{gathered}
$$

and:

$$
\mathbb{F}_{e}^{\prime}=\frac{d}{d X}\left[\left(\begin{array}{l}
f_{e} \\
c_{e}
\end{array}\right)-\left(\begin{array}{l}
f_{e a} \\
c_{e a}
\end{array}\right)\right]
$$

$$
=\left(\begin{array}{ll}
1 & 0 \\
\hat{r}_{e} & 1
\end{array}\right)\left(\begin{array}{c}
\left(\rho A+c_{l m}\right) \gamma_{e} \\
\left(\rho I+c_{a m}\right) \dot{\omega}_{e}+\omega \times(\rho I \omega)
\end{array}\right)
$$

and finally:

$$
\mathbb{F}_{d l}^{\prime}=\left(\begin{array}{cc}
1 & 0 \\
\hat{r}_{e} & 1
\end{array}\right)\left(\begin{array}{l}
d f_{d r a g} / d X \\
d c_{d r a g} / d X
\end{array}\right)
$$

where II denotes inertia and added mass tensor, $-\mathbb{F}_{e}$ is the wrench of Coriolis-centrifugal and strain forces produced by material and added mass, and $\mathbb{F}_{d l}$ is the wrench generated by the local drag forces and torques applied along the eel. And where (54) and (56) are initialized by the following boundary conditions compatible with (44) and (16), with $c_{m, \text { head }}=C_{m, \text { head }} t_{1 o} \otimes t_{1 o}:$

$$
\mathbb{I}(0)=\left(\begin{array}{cc}
c_{m, \text { head }} & 0 \\
0 & 0
\end{array}\right), \quad \mathbb{F}_{d l}(0)=\left(\begin{array}{c}
f_{d, \text { head }} \\
0
\end{array}\right)
$$

While:

$$
\mathbb{F}_{e}(0)=\left(\begin{array}{l}
0 \\
0
\end{array}\right)
$$

For simulation purpose, (54)-(56) are projected into the head frame. Finally, once the equation (48) replaced by its parameterization in terms of a quaternion field (noted $q$ ), all the equations from (48) to (57) can be solved by integrating the following first order system:

$$
x_{1}^{\prime}=f_{1}\left(x_{1}, K_{d}(X, t), \dot{K}_{d}(X, t), \ddot{K}_{d}(X, t)\right), x_{1}(X=0)=x_{1 o}
$$

where:

$$
x_{1}^{T}(X)=\left(r^{T}, q^{T}, \dot{r}^{T}, \omega^{T}, \gamma_{e}^{T}, \dot{\omega}_{e}^{T}, \operatorname{vect}(\mathbb{I})^{T}, \mathbb{F}_{e}^{T}, \mathbb{F}_{d l}^{T}\right),
$$

and:

$$
x_{1 o}^{T}=\left(r_{o}^{T}, q_{o}^{T}, \dot{r}_{o}^{T}, \omega_{o}^{T}, 0,0, \operatorname{vect}(\mathbb{I}(0))^{T}, 0, \mathbb{F}_{d l}^{T}(0)\right)
$$

with vect $(I)$, the $6 \times 1$ vector of the independent components of the tensor $\mathbb{I}$; and $\mathbb{I}(0), \mathbb{F}_{d l}(0)$ given by (57). At the end, from this first space loop we obtain the head acceleration $\left(\ddot{r}_{o}, \dot{\omega}_{o}\right)$ from:

$$
\left(\begin{array}{c}
\ddot{r}_{o} \\
\dot{\omega}_{o}
\end{array}\right)=\mathbb{I}^{-1}\left(\mathbb{F}_{d l}-\mathbb{F}_{e}\right),
$$

which initializes the second space loop.

\section{B. Second space integration loop}


From the following o.d.e.'s:

$$
\begin{gathered}
\dot{\omega}^{\prime}=R \ddot{K}_{d}+\omega \times R \dot{K}_{d}, \dot{\omega}(0)=\dot{\omega}_{o} \\
\ddot{r}^{\prime}=\dot{\omega} \times r^{\prime}+\omega \times\left(\omega \times r^{\prime}\right), \ddot{r}(0)=\ddot{r}_{o}
\end{gathered}
$$

we recover the fields of Galilean acceleration of the eel's body. Then inserting $\ddot{r}$ into the first p.d.e. of (42) gives:

$$
n^{\prime}=-\left(\rho A-c_{l m}\right) \ddot{r}+d f_{d r a g} / d X
$$

Next integrating (63) in relation to $X$ at each step time, gives the field of internal forces applied to the vertebrae. At this step, inserting $n$ and $\dot{\omega}$ in the second p.d.e. of (42) and integrating :

$$
\begin{gathered}
M^{\prime}+K_{d} \times M=-R^{T}\left(\left(\rho I-c_{a m}\right) \dot{\omega}+\omega \times(\rho I \omega)\right) \\
-R^{T}\left(t_{1} \times n-d c_{d r a g} / d X\right)
\end{gathered}
$$

with respect to $X$, gives the field of control torque: $t \mapsto M_{d}(X, t), \forall X, \quad$ required to force the expected curvature-twist law: $t \mapsto K_{d}(X, t), \forall X$.

Finally, once completed by (48)-(51), (61)-(64) are solved by integrating the following first order system:

$$
x_{2}^{\prime}=f_{2}\left(x_{2}, K_{d}(X, t), \dot{K}_{d}(X, t), \ddot{K}_{d}(X, t)\right)
$$

where:

$$
x_{2}^{T}(X)=\left(r^{T}, q^{T}, \dot{r}^{T}, \omega^{T}, \ddot{r}^{T}, \dot{\omega}^{T}, n^{T}, M^{T}\right),
$$

and:

$$
x_{2 o}^{T}=\left(r_{o}^{T}, q_{o}^{T}, \dot{r}_{o}^{T}, \omega_{o}^{T}, \ddot{r}_{o}^{T}, \dot{\omega}_{o}^{T}, n^{T}(0), 0\right)
$$

with $n(0)$, given by (44).

Once the second space loop is completed, the head acceleration is time-integrated twice over in order to update the state of the head. Then the time is shifted by one step and the algorithm resumes...

\section{Remark:}

Let us note that (63) and (64) play the role of the backward recursive equations of inter-body wrenches of a serial manipulator [15]. Nevertheless, contrary to the case of a manipulator, where the boundary conditions on wrenches are known at the end of the structure and are unknown at the base, in our case we know the boundary wrenches at both ends, so forward or backward integration is equivalent. In the following, we choose to adopt a forward recursive equation on wrenches initialized by (44). It follows that the second boundary condition (45) will be a verification test.

\section{NUMERICAL RESULTS}

In this section, we report some numerical results obtained by the algorithm of section VIII. The objective of these examples is to prove that despite the non-linear character of the eel dynamics we can easily realise three-dimensional gaits by combining some elementary curvature laws, while maintaining the twist at zero. Before presenting the numerical results, let us describe the robot characteristics.

\section{A. Geometric description of the eel-like prototype:}

In order to take into account the continuous property of the skin of the prototype, we consider the previous macroscopic modeling with the following geometry (see Figure 3):

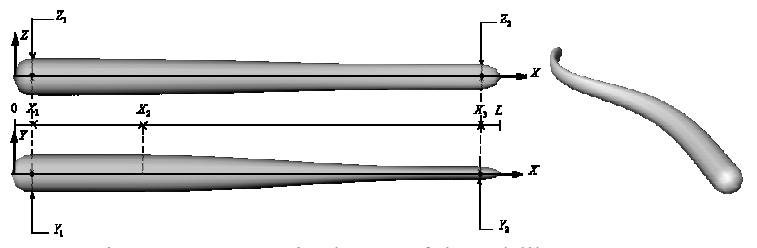

Figure 3: Geometric shape qof the eel-like prototype,

where the total length of the robot is of $L=2$ meters, its total mass is $21,7 \mathrm{~kg}$, its density is 1 . The cross section is of elliptic shape (of great axis maximal length equal to $15 \mathrm{~cm}$ ) on the interval $\left[X_{1}, X_{3}\right]$, while the head $\left(X \in\left[X_{o}, X_{1}\right]\right)$ and the tail $\left(X \in\left[X_{3}, L\right]\right)$ are respectively half of a sphere (of a diameter of $15 \mathrm{~cm}$ ), and that of an ellipsoid (of great and small axis length equal to $10 \mathrm{~cm}$ and $5 \mathrm{~cm}$, respectively). Finally, denoting the small and great axis' lengths of the elliptic section by $b$ and $c$, the model (9)-(13), is used with:

$$
\begin{aligned}
& C_{l d 1}=(1 / 2) \rho C_{1} \pi(b+c) / 2, C_{l d 2}=(1 / 2) \rho C_{2} 2 c, \\
& C_{l d 3}=(1 / 2) \rho C_{3} 2 b, C_{a d 1}=(1 / 2) \rho C_{4}\left(b^{2}-c^{2}\right)^{2}, \\
& C_{l m 2}=\rho \pi c^{2} C_{5}, C_{l m 3}=\rho \pi b^{2} C_{6}, C_{a m 1}=\rho(\pi / 8)\left(c^{2}-b^{2}\right)^{2} C_{7},
\end{aligned}
$$

with from [28,32], $C_{1}=0.01, \quad C_{5}=C_{6}=C_{7}=1$, and $C_{2}=C_{3}=C_{4}=1$; which correspond to the values of a cylindrical obstacle plunged in a flow with a Reynolds of approximately: $R_{e} \simeq 10^{5}$, i.e. an eel velocity of $1 \mathrm{~ms}^{-1}$ approximately.

\section{B. First example: Nominal planar propulsion:}

Following the standard uses of biomechanics literature about anguilliform locomotion [33,34], we started our numerical investigations with planar forward propulsion. This planar motion is produced by a curvature law of the following form:

$$
K_{1}=K_{2}=0, K_{3}=\alpha(X) h(t),
$$

with $\alpha$ given in terms of $X$, by: $\alpha=\alpha_{1} X^{2}+\alpha_{2} X+\alpha_{3}$, where the $\alpha_{i}$ 's are arbitrary constant coefficients. The time varying law $h$ is defined by:

$$
h(X, t)=h_{o}(t) h_{1}(X, t),
$$

where $h_{o}$ is a starting function that allows the eel to start smoothly from rest to nominal propulsion mode: 


$$
h_{o} /\left\{\begin{array}{l}
h_{o}(t)=0, \text { for: } t<t_{i} \\
h_{o}(t)=\left(\left(t-t_{i}\right) /\left(t_{f}-t_{i}\right)\right) \\
-(1 /(2 \pi)) \sin \left(\left(t-t_{i}\right) /\left(t_{f}-t_{i}\right)\right) \\
\text { for: } t_{i} \leq t \leq t_{f} \\
h_{o}(t)=1, \text { for: } t>t_{f}
\end{array}\right.
$$

This term multiplies the propulsion term, inspired by the experimental observation of the animal [33]:

$$
h_{1}(X, t)=\sin \left[2 \pi\left(\frac{X}{\lambda}-\frac{t}{T}\right)\right]
$$

This second term represents the propagation of curvature waves from the head to the tail with a constant time frequency $1 / T$ and wavelength $\lambda$. Simulation is achieved with the following numerical values: $\alpha_{1}=1, \alpha_{2}=-0.75$, $\alpha_{3}=1, t_{i}=0 s, t_{f}=1 s, \lambda=1 \mathrm{~m}, T=1 \mathrm{~s}$. In Figure 4 we report several configurations of the eel in the plane obtained every $1.25 \mathrm{sec}$., while Figure 5 represents the time evolution of the eel's head twist in the earth frame.

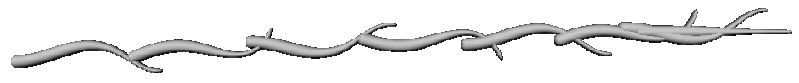

Figure 4: Eel's configuration every $1.25 \mathrm{sec}$.

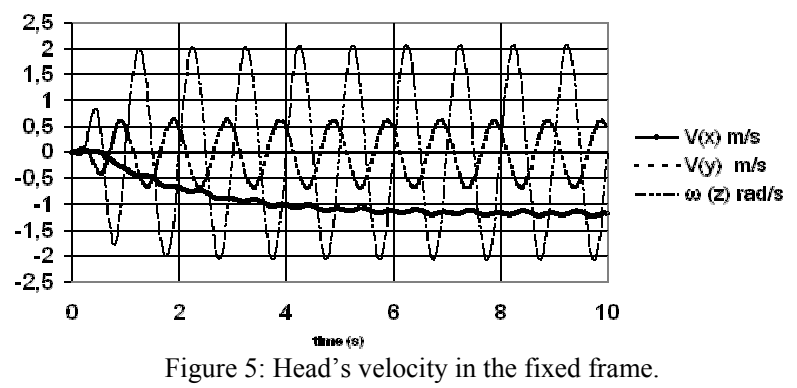

\section{Second example: Plane turning law:}

In order to make the eel turn in the plane (Figure 6), we add a constant offset to the previous propulsion term. This is the continuous version of the joint law adopted in [4]. The constant curvature offset is imposed progressively through the starting law (68). Hence, the curvature law is now:

$$
K_{1}=K_{2}=0, K_{3}=\alpha(X) h(t)+K_{3, o f} h_{o}(t) .
$$

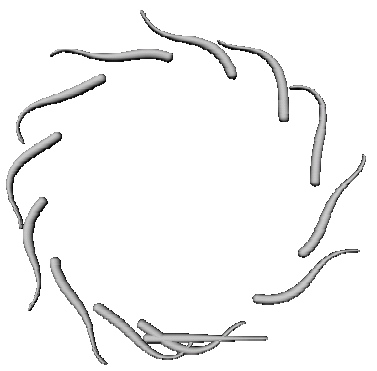

Figure 6: Eel's configuration every $1.25 \mathrm{sec}$.

\section{Third example: Submergence}

The goal of this example is to achieve submergence from one given altitude to another (see Figure 7). This is accomplished by adding to the propulsive law of the first test, a curvature law $t \mapsto K_{2}(t)$ around the second axis of the eel section frames. Hence the resultant curvature law is:

$$
K_{1}=0, K_{2}=K_{2}(t), K_{3}=\alpha(X) h(t),
$$

with:

$$
\begin{aligned}
& \left\{\begin{array}{l}
K_{2}(t)=0, \text { for: } t<t_{1} \\
K_{2}(t)=P\left(t_{1}, t_{2}, 0, K_{2 o f f}\right), \text { for: } t_{1} \leq t<t_{2} \\
K_{2}(t)=P\left(t_{2}, t_{3}, K_{2 o f f}, 0\right), \text { for: } t_{2} \leq t<t_{3} \\
K_{2}(t)=0, \text { for: } t_{2} \leq t<t_{3}
\end{array}\right. \text { and : } \\
& \left\{\begin{array}{l}
K_{2}(t)=P\left(t_{4}, t_{5}, 0,-K_{2 \text { off }}\right), \text { for: } t_{4} \leq t<t_{5} \\
K_{2}(t)=P\left(t_{5}, t_{6},-K_{2 o f f}, 0\right), \text { for: } t_{5} \leq t<t_{6}, \\
K_{2}(t)=0, \text { for: } t \geq t_{6}
\end{array}\right.
\end{aligned}
$$

where $P\left(t_{i}, t_{j}, f_{i}, f_{j}\right)$ is a fifth order polynomial that interpolates $f$ from $f\left(t_{i}\right)=f_{i}$ to $f\left(t_{j}\right)=f_{j}$ with first and second order derivatives equal to zero, while guaranteeing a second order time continuity.

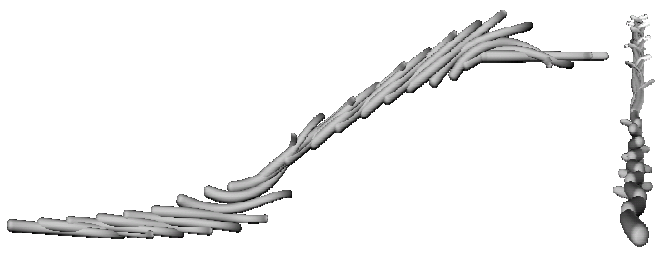

Figure 7: Eel's configuration obtained every $0.5 \mathrm{sec}$.

Lastly, we surmised a three-dimensional plunging following a spiral. This is achieved using the following curvature law, which superimposes the turning curvature law of example 2 with, a $K_{2}$ 's time evolution given by: $K_{2}=K_{2, o f .} h_{o}(t)$, where $h_{o}$ is the starting time function.

$$
K_{1}=0, K_{2}=K_{2, o f .} h_{o}, K_{3}=h_{o}(t)\left(\alpha(X) h_{1}(X, t)+K_{3, o f}\right) .
$$

Figure 8 shows the results of this test with: $K_{2, o f .}=$ $K_{3, o f .}=0.5$ :
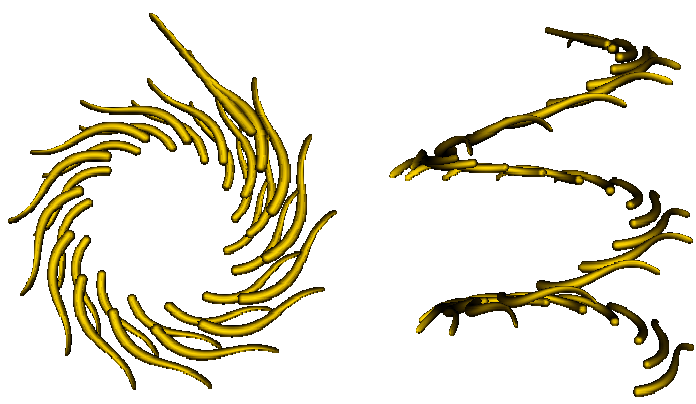

Figure 8: Eel's configuration every $1 \mathrm{sec}$. . 
The Figure 9 give the time evolutions of the three components of the control torque field $M$ evaluated at $X=L / 2=1 m$.
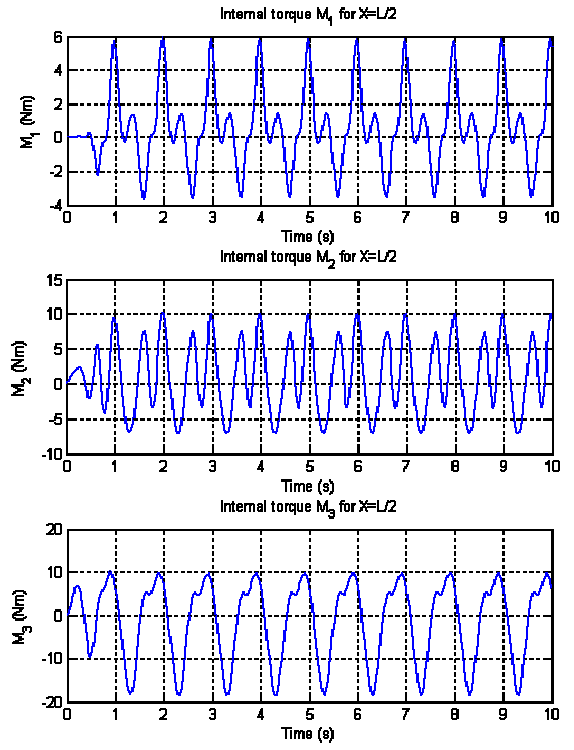

Figure 9: Time evolution of the internal torque for the section at $X=1 \mathrm{~m}$ in its mobile frame.

Finally, Figure 10 validates the whole dynamic balance of the eel. The computation of internal force $n$ and torque $M$ is computed all over the back-bone (here at $t=10 \mathrm{~s}$ ) using a forward space integration and we obtain $n(L)=M(L)=0$ as pointed out in the remark of section VIII.
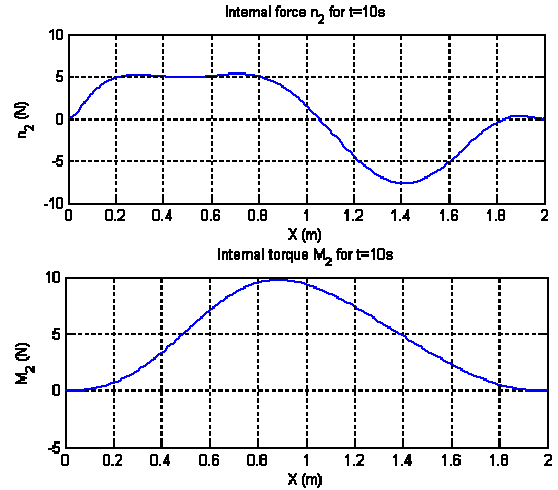

Figure 10: $X$ profile of the second component of the internal force and torque at $t=10 \mathrm{~s}$ (in the section frame).

\section{CONCLUSION}

This paper presents a dynamic model of a swimming eellike robot. Contrary to the prior results about the biomimetic robotics of the eel, the proposed solution to the dynamics is capable of modeling an ideal three-dimensional continuous prototype. The design of the prototype will be based on the serial assembly of many parallel platforms of "spherical joint type", and the multi-body system will be covered with a continuous flexible organ copying the eel's skin. The continuous approach has the advantage of providing a macroscopic model of the prototype, and in particular giving an overview of swimming dynamics without entering into the details of the modeling of complex internal hybrid (parallel and serial) kinematics and their dynamics. This advantage has already been tested in the framework of our project. Macro-continuous modeling allows us to rapidly investigate the locomotion and the control of the ideal prototype. Moreover it is extensively used today to assist in designing the future prototype. In order to get an exact model (from kinematics to dynamics), we based our development on the "geometrically exact theory" of nonlinear beams, a new promising paradigm of non-linear structural mechanics. Based on the assumption of Cosserat medium, this theory gives an exact model of finite rotations of some rigid "micro-solids" right from the beginning of the analysis. In our case, these micro-solids are the beam cross sections copying the vertebrae of the fish. This choice, allows us to obtain a three dimensional dynamic model which will be used in the future to control three-dimensional swimming, a problem to our knowledge never investigated in robotics. Moreover, based on the literature of fluid mechanics, a simplified, but quite complete model of the fluid-structure contact is adopted. The geometrically exact approach is developed in the Newton-Euler formalism, as it is well known among the robotics dynamics community. Based on these modeling choices, the proposed algorithm allows one to compute the motion of the eel and the control torque distribution from the knowledge of the desired internal deformation imposed to its body. Finally, this algorithm constitutes a generalization of the computed torque approach of articulated manipulators. The generalization here concerns the continuous character of the hyper-redundant robot's model and also the mobility of the base, which plays the role of the eel's head.

Acknowledgment: We would like to thank the French CNRS for supporting this work through the project "Robot Anguille" of the Interdisciplinary Research Program ROBEA. We acknowledge the constructive remarks of the reviewers, which have contributed to improve the quality of this paper.

\section{REFERENCE}

[1] M. S. Triantafyllou, G. S. Triantafyllou and R. Gopalkrishnan, "Optimal Thrust Development in Oscillating Foils with Application to Fish Propulsion", J. Fluids Structures, vol. 7, pp. 205-224, 1993.

[2] R. Mason and J.W. Burdick, "Experiments in Caranguiform Robotic Fish Locomotion", IEEE Int. Conf. Robotics and Automation, San Francisco, 2000, pp. 428-435.

[3] K.A. Morgansen, P.A. Vela and J.W. Burdick, "Trajectory Stabilization for a Planar Caranguiform Robot Fish", IEEE Int. Conf. Robotics and Automation, Washington, 2002, pp. 756-762.

[4] K.A. McIsaac and J.P. Ostrowski, "A Geometric Approach to Anguilliform Locomotion Modelling of an Underwater Eel Robot", IEEE Int. Conf. Robotics and Automation, Detroit, 1999, pp. 2843-2848.

[5] N. Kato and T. Inaba, "Guidance and Control of Fish Robot with Apparatus of Pectoral Fin Motion", IEEE Int. Conf. Robotics and Automation, Leuven, 1998, pp. 446-451.

[6] G.S. Chirikjan and J.W. Burdick, "An Obstacle Avoidance for HyperRedundant Manipulators", IEEE Int. Conf. Robotics and Automation, Cincinnati, 1990, pp. 14-17.

[7] G.S. Chirikjian and J.W. Burdick, "The Kinematics of HyperRedundant Robot Locomotion", IEEE Transactions on Robotics and Automation, vol. 11, no.6, Nagoya, 1995, pp. 781-793.

[8] H. Choset and W. Henning, "A Follow-The-Leader Approach to Serpentine Robot Motion Planning", ASCE J. of Aerospace Engineering, vol. 12, no. 2, pp.65-73, 1999. 
[9] J.C. Simo, "A Finite Strain Beam Formulation. The Three-Dimensional Dynamic Problem. Part I: Formulation and Optimal Parametrization ", Comp. Meth. Appl. Mech. Eng., vol. 72, pp. 267-304, 1989.

[10] J.C. Simo and L. Vu-Quoc, "A Three-Dimensional Finite-Strain Rod Model. Part II: Computational Aspects", Comp. Meth. Appl. Mech. Eng., vol. 58, pp. 79-116, 1986.

[11] J.C. Simo and L. Vu-Quoc, "On the Dynamics in Space of Rods Undergoing Large Motions - A Geometrically Exact Approach", Comp. Meth. Appl. Mech. Eng., vol. 66, pp. 125-161, 1988.

[12] J.P. Ostrowski and J.W. Burdick, "The Geometric Mechanics of Undulatory Robotics Locomotion", The International Journal of Robotics Research, vol.17, no.7, pp.683-701, 1998.

[13] J.P. Ostrowski and al., "The Mechanics of Undulatory Locomotion: the Mixed Kinematic and Dynamic Case", IEEE International Conference on Robotics and Automation, vol. 2, Nagoya, 1995, pp.1945-1951.

[14] D.K. Kelly and R.M. Murray, "Geometric Phases and Robotic Locomotion”, Journal of Robotic Systems, vol. 12, no. 6, pp. 417-431, 1995.

[15] J.Y.S. Luh, M.W. Walker and R.C.P. Paul, "On-Line Computational Scheme for Mechanical Manipulator", Trans. of ASME, J. of Dynamic Systems, Measurement, and Control, vol. 102, no.2, pp.69-76, 1980.

[16] W. Khalil and E. Dombre, Modeling, Identification and Control of Robots. London : Penton-Sciences, 2002.

[17]R. Featherstone, "The Calculation of Robot Dynamics Using Articulated-Body Inertias", Int. Journ. of Robotics Research, vol. 2, no. 1, pp. 13-30, 1983

[18] J. Katz and A. Plotkin, "Low Speed Aerodynamics". Cambridge Univ. Press, secd. ed., 2002.

[19] J. Lighthill, "Mathematical Biofluid-dynamics". Philadelphia: SIAM (book), 1973.

[20] J.J. Burgess, "Bending Stiffness in a Simulation of Undersea Cable Deployment", Intern. Journal of Offshore and Polar Engineering, vol. 3, no.3, 1993.

[21]E. and F. Cosserat, Théorie des corps déformables. Paris : Hermann, 1909 (in French).

[22]E. Reissner, "On a One-Dimensional Large Displacement FiniteStrain Theory". Stud. Appl. Math., vol. 52, pp. 87-95,1973.

[23] J.P. Merlet, Parallel Robots. Kluwer Academic Publishers, 2000.

[24] S.P. Timoshenko, Théorie Elémentaire et Problèmes. Paris: Dunod, 1968 (in French).

[25]F. Boyer and D. Primault, "Finite Element of Slender Beams in Finite Transformations - a Geometrically Exact Approach", International Journal of Numerical Methods in Engineering, vol. 59, no. 5, pp. 669702, 2004.

[26] J.R. Morison and al., "The Force Exerted by Surface Waves on Piles", Transaction of the AIME, vol. 189, pp.149-154, 1950.

[27] A.H. Techet and M.S. Triantafyllou, "Fluid Forces on Bodies", Lecture Course for Ocean Engineering. MIT, 2004.

[28] G. Susbielles and Ch. Bratu, Vagues et Ouvrages Pétroliers en Mer. Paris : Editions Technip, 1981 (in french).

[29] A. Cardona and M. Géradin, "A Beam Finite Element Non-Linear Theory with Finite Rotations". Int. J. Numer. Meth. Engng., vol. 26, 2403-2438, 1988.

[30]H. Goldstein, Classical Mechanics. Addison-Wesley, Reading, MA, 1980.

[31] L. Meirovitch, Dynamics and Control of Structures. New-York: John Wiley \& Sons Ed., 1990.

[32] R. Comolet, Mécanique Expérimentale des Fluides. Paris: Masson, 1994 (in French).

[33] G.B. Gillis, "Environmental Effects on Undulatory Locomotion in the American Eel Anguilla Rostrata: Kinematics in Water and on Land", J. of Experimental Biology, vol. 201, pp.949-961, 1998.

[34] U.K. Müller \& al, "How Body Contributes to the Wake in Undulatory Fish Swimming: Flow Fields of Swimming Eel (Anguilla Anguilla)", J. of Experimental Biology, vol. 204, pp. 2751-2762, 2001.
The purpose of this Appendix is to show that in the case of an eel's circular cross section, the basic model (9) and (11), exactly reduces to the model of fluid forces of circular cross sectional cable of [20]. As a matter of fact, introducing the coefficients of section IX.A into (9) and (11) (with $P$ the cross section perimeter) first gives:

$$
\begin{gathered}
d f_{\text {ext }} / d X=-\frac{1}{2}\left(\rho C_{1} P\left|V_{1}\right| V_{1}\right) t_{1}-\frac{1}{2} \rho\left(C_{2} 2 c\left\|v_{n}\right\| V_{2}+2 C_{5} \pi c^{2} \Upsilon_{2}\right) t_{2} \\
-\frac{1}{2} \rho\left(C_{3} 2 b\left\|v_{n}\right\| V_{3}+2 C_{6} \pi b^{2} \Upsilon_{3}\right) t_{3}, \\
d c_{e x t} / d X=-\frac{1}{2} \rho\left(C_{4}\left(b^{2}-c^{2}\right)^{2}\left|\Omega_{1}\right| \Omega_{1}\right) t_{1}-\frac{1}{8} \rho\left(C_{7} \pi\left(c^{2}-b^{2}\right)^{2} \dot{\Omega}_{1}\right) t_{1} .
\end{gathered}
$$

Then, let us consider a circular cross section by making:

- $2 b=2 c=D:$ diameter of the circular cross section,

- $\quad P=\pi D:$ perimeter of the circular cross section

- $\pi b^{2}=\pi c^{2}=A:$ cross section area,

- $C_{2}=C_{3}=C_{n}$ : normal drag coefficient of the circular cross section,

- $C_{5}=C_{6}=C_{m}$ : added mass coefficient of the circular cross section,

gives:

$$
\begin{gathered}
d f_{\text {ext }} / d X=-\frac{1}{2}\left(\rho C_{t} \pi D\left|V_{1}\right| V_{1}\right) t_{1}-\frac{1}{2} \rho\left(C_{n} D\left\|v_{n}\right\| V_{2}+2 C_{m} A \Upsilon_{2}\right) t_{2} \\
-\frac{1}{2} \rho\left(C_{n} D\left\|v_{n}\right\| V_{3}+2 C_{m} A \Upsilon_{3}\right) t_{3}, \\
d c_{e x t} / d X=0,
\end{gathered}
$$

which is quite simply the model of [20] with $C_{t}=C_{1}$ : the "tangential drag coefficient".

\section{APPENDIX}

